\title{
Vagal nerve stimulation prevents reperfusion injury through inhibition of opening of mitochondrial permeability transition pore independent of the bradycardiac effect
}

Rajesh G. Katare, MD, ${ }^{\text {a,* }}$ Motonori Ando, PhD, ${ }^{\text {a,c }}$ Yoshihiko Kakinuma, MD, ${ }^{a}$ Mikihiko Arikawa, PhD, ${ }^{\text {a }}$ Takemi Handa, MD, Fumiyasu Yamasaki, MD, ${ }^{\mathrm{b}}$ and Takayuki Sato, $\mathrm{MD}^{\mathrm{a}}$

Background: In spite of recent advances in coronary interventional therapy, reperfusion injury is still considered to be a major problem in patients undergoing surgical procedures, such as bypass grafting. Here we demonstrate a novel therapeutic strategy against ischemia-reperfusion injury: vagally mediated prevention of reperfusioninduced opening of mitochondrial permeability transition pore.

Methods: We investigated the effects of efferent vagal stimulation on myocardial reperfusion injury with ex vivo and in vitro rat models. In the ex vivo model the hearts were perfused with intact vagal innervation, which allowed us to study the effects of the vagal nerve on the heart without other systemic effects.

Results: Compared with sham stimulation, vagal stimulation exerted a marked anti-infarct effect irrespective of the heart rate $(34 \% \pm 6 \%$ vs $85 \% \pm 9 \%$ at a heart rate of 300 beats $/ \mathrm{min}, 37 \% \pm 4 \%$ vs $43 \% \pm 5 \%$ at a heart rate of 250 beats $/ \mathrm{min}$, and $39 \% \pm 4 \%$ vs $88 \% \pm 7 \%$ at a heart rate of 350 beats $/ \mathrm{min}$ ) after a 30 -minute period of global ischemia, activated cell-survival Akt cascade, prevented downregulation of the antiapoptotic protein Bcl-2, and suppressed cytochrome-c release and caspase-3 activation. Furthermore, vagal stimulation-treated hearts exhibited a significant improvement in left ventricular developed pressure (78 $\pm 5 \mathrm{vs} 45 \pm 8 \mathrm{~mm} \mathrm{Hg}$ ) and a significant attenuation in an incremental change in left ventricular end-diastolic pressure during reperfusion. These beneficial effects of vagal stimulation were abolished by a permeability transition pore opener, atractyloside. In the in vitro study with primary-cultured cardiomyocytes, acetylcholine prevented a reoxygenation-induced collapse in mitochondrial transmembrane potential through inhibition of permeability transition pore opening.

Conclusion: Vagal stimulation would be a potential adjuvant therapy for the rescue of ischemic myocardium from reperfusion injury, and the protective effects are independent of its bradycardiac effects.

Although reperfusion or restoration of blood flow to ischemic myocardium is necessary for salvage of cardiac cells and function, reperfusion itself initiates a cascade of events that results in cardiac cell death and dysfunction. ${ }^{1,2}$ Therefore reperfusion injury is still considered a major problem in patients with acute coronary occlusion or those undergoing surgical procedures, such as coronary artery bypass grafting $(\mathrm{CABG})$ and transplantation. Mitochondria are the cell's powerhouse, the site where the vast majority of adenosine triphosphate (ATP) is synthesized; under pathologic

\footnotetext{
From the Departments of Cardiovascular Control $^{\mathrm{a}}$ and Clinical Laboratory, ${ }^{\mathrm{b}}$ Kochi Medical School, Nankoku, Japan, and the Department of Science and Education, ${ }^{c}$ Okayama University, Okayama, Japan.

* Dr Katare is currently affiliated with the Bristol Heart Institute, University of Bristol, Bristol, United Kingdom.

Supported by a Health and Labor Sciences Research Grant (H14-NANO-002, H16NANO-005) from the Ministry of Health, Labor, and Welfare of Japan and by a Grant-in-Aid for Scientific Research $(15300165,17790892)$ from the Ministry of Education, Science, Sports, and Culture of Japan.

Received for publication Sept 20, 2007; revisions received July 16, 2008; accepted for publication Aug 8, 2008.

Address for reprints: Takayuki Sato, MD, Department of Cardiovascular Control, Kochi Medical School, Nankoku, Kochi 783-8505, Japan (E-mail: tacsato-kochimed@ umin.ac.jp).

J Thorac Cardiovasc Surg 2009; 137:223-31

$0022-5223 / \$ 36.00$

Copyright (c) 2009 by The American Association for Thoracic Surgery

doi:10.1016/j.jtcvs.2008.08.020
}

conditions, however, mitochondria often play a decisive role in cell death. Mitochondrial decision of cellular life or death is closely linked to the electrochemical gradient built across the inner mitochondrial membrane that is nearly impermeable to all ions, including protons. As one of the crucial mechanisms for reperfusion injury, well known is the reperfusion-related opening of a nonspecific pore in the inner mitochondrial membrane: the permeability transition pore (PTP). The prolonged opening of the PTP leads cells to death through rapid depletion of ATP and activation of death messengers, such as caspases. ${ }^{3}$

Our previous study ${ }^{4}$ demonstrated that efferent vagal stimulation (VS), when administered before or even during 30-minute coronary occlusion, protects hearts against ischemia-induced lethal arrhythmias through prevention of the loss of functional gap-junction channels. In another study ${ }^{5}$ we examined the effect of VS on ischemic insult during 3hour coronary occlusion, showing that VS significantly reduced infarct size through activation of phosphatidylinositol 3-kinase and Akt. With these in mind, we speculated that a brief period of VS, before or during acute ischemia, would be expected to suppress lethal arrhythmias at an early phase of acute coronary occlusion and to reduce ischemic insult during long-lasting occlusion. However, the effect of VS on reperfusion injury still remains to be elucidated. 


$$
\begin{aligned}
& \text { Abbreviations and Acronyms } \\
& \begin{aligned}
\text { ACh } & =\text { acetylcholine } \\
\text { ATP } & =\text { adenosine triphosphate } \\
\text { CABG } & =\text { coronary artery bypass grafting } \\
\text { PTP } & =\text { permeability transition pore } \\
\text { SS } & =\text { sham stimulation } \\
\text { VS } & =\text { vagal stimulation }
\end{aligned}
\end{aligned}
$$

Since Murry and colleagues ${ }^{6}$ reported an endogenous protective mechanism against reperfusion-related injury (ie, ischemic preconditioning), numerous studies have been performed to elucidate its cellular and molecular mechanisms. A series of detailed experimental studies by Downey and colleagues ${ }^{7,8}$ has shown that several drugs, such as bradykinin and acetylcholine ( $\mathrm{ACh}$ ), mimic ischemic preconditioning through a complex pathway, including activation of phosphatidylinositol 3-kinase and Akt, suggesting that these drugs are potent preconditioning agents. Although ischemic or pharmacologic preconditioning therapy has been available for more than a decade to patients undergoing CABG, its application for patients presenting with acute myocardial infarction is quite difficult. The primary reason is that, by definition, the preconditioning intervention must be administered before the onset of acute myocardial infarction. The cardioprotective intervention that can be performed during coronary occlusion has therefore been expected for years, as has postconditioning therapy at reperfusion. ${ }^{9,10}$ Based on these clinical backgrounds and recent findings that efferent VS, when administered even during acute coronary occlusion, increases ACh concentration in ventricular interstitial fluid ${ }^{11}$ and that Akt contributes toward inhibiting the reperfusion-related opening of the PTP, ${ }^{10,12}$ we hypothesized that VS during coronary occlusion could exert a cardioprotective effect at reperfusion by targeting the PTP. In the present study we tested this hypothesis with ex vivo and cellular models of acute myocardial ischemia and reperfusion and thus propose a new therapeutic paradigm, neurally mediated conditioning during coronary occlusion, as well as preconditioning before occlusion and postconditioning at reperfusion.

\section{MATERIALS AND METHODS}

Male Wistar rats (SLC, Shizuoka, Japan) aged between 8 and 10 weeks and weighing $250 \pm 20 \mathrm{~g}$ and 2-day-old neonatal rats were used. All animals received humane care in compliance with the "Guide for the care and use of laboratory animals" prepared by the Institute of Laboratory Animal Resources and published by the National Institutes of Health (NIH publication no. 86-23, revised 1986).

\section{Myocardial Ischemia-Reperfusion Models}

We examined the effects of efferent VS on ischemia-reperfusion injury with ex vivo and cellular models of acute myocardial ischemia-reperfusion. The main aim of the ex vivo study was to clarify the effects of VS on iso- volumetric left ventricular function and subcellular signaling pathways of reperfused hearts in the absence of extracardiac regulatory systems, such as baroreflex or the sympathoadrenal system, and thus such a simplified preparation could exclude any effects of these extracardiac regulatory mechanisms on cardiac and subcellular functions during reperfusion. Moreover, to mimic the ischemia-reperfusion heart injury at the cellular level and to clarify the direct action of efferent VS, we investigated the effect of ACh, a neurotransmitter that is released at cardiac nerve endings by VS, on hypoxia-reoxygenation cell injury.

\section{Ex Vivo Model With Vagal Innervation}

To study the effect of VS on the recovery of cardiac function during reperfusion, we used a global ischemia-reperfusion model with an intact vagal innervation. After the ascending aorta was cannulated, the heart of the adult rat was in situ perfused in a Langendorff apparatus with filtered Krebs-Henseleit buffer equilibrated with $5 \%$ carbon dioxide and $95 \%$ oxygen. To preserve vagal innervation of the heart, we cut bilateral vagal nerves at the cervical level and then excised the heart en bloc with the trachea and esophagus, as described previously. ${ }^{13}$ A fluid-filled latex balloon was passed into the left ventricle through the mitral valve and connected to the pressure transducer for continuous monitoring of left ventricular pressure. The balloon was inflated to set an end-diastolic pressure of $10 \mathrm{~mm}$ $\mathrm{Hg}$. Coronary perfusion pressure was controlled at $80 \mathrm{~mm} \mathrm{Hg}$, and coronary flow was measured continuously.

To study the effects of VS on reperfusion injury, the right vagal nerve was isolated, and the proximal portion was cut to exclude the effects of vagal afferent. The efferent portion was then placed on a pair of platinum wires and stimulated with an isolated constant voltage stimulator (SS-202J and SEN-7203; Nihon Kohden, Tokyo, Japan) with continuous electrical rectangular pulses of $0.1 \mathrm{~ms}$ in duration at $10 \mathrm{~Hz}$. The electrical voltage of pulses was optimized in each rat so as to obtain a $10 \%$ reduction in heart rate before ischemia. We confirmed that a bradycardiac response of the Langendorffperfused heart to VS was stably reproducible for 90 minutes after the beginning of perfusion (unpublished observation). Hearts were paced at 3 different rates $(250,300$, and 350 beats/min) throughout the experiment to demonstrate the heart rate-independent effects of VS in protecting the ischemic myocardium.

The heart was subjected to 30 minutes of global ischemia by stopping the perfusion of Krebs-Henseleit buffer, followed by 120 minutes of reperfusion. Sham stimulation (SS) or VS was administered starting 5 minutes before ischemia and continuing for 30 minutes of reperfusion. Left ventricular pressure and coronary flow were digitally recorded with a laboratory computer and later analyzed with Igor-Pro version 3.1 (WaveMetrics, Lake Oswego, Ore). To examine the effect of PTP opening on the hearts treated with SS and VS, we added atractyloside ${ }^{14}(0.02 \mathrm{mmol} / \mathrm{L}$; Sigma, St Louis, Mo) to perfusate before ischemia. Ten animals were studied in each group of experiments.

\section{In Vitro Cellular Model and ACh Treatment}

Neonatal cardiomyocytes were isolated as described previously. ${ }^{4}$ After 4 days of culture, the cells were subjected to chemically induced hypoxia for 60 minutes with cobalt chloride $(0.1 \mathrm{mmol} / \mathrm{L})$ and then reoxygenated for 180 minutes. The effects of ACh $(0.5 \mathrm{mmol} / \mathrm{L})$, atropine $(0.1 \mathrm{mmol} / \mathrm{L})$, and atractyloside $(0.02 \mathrm{mmol} / \mathrm{L})$ were evaluated.

\section{Infarct Size Assessment and MTT Assay of Cell Viability}

In both in vivo and ex vivo studies, the infarcted area was determined by using a triphenyltetrazolium chloride staining method, as described previously. ${ }^{4}$

To evaluate the effect of ACh on the viability of hypoxia-reoxygenated cardiomyocytes, we used a colorimetric method with an MTT Cell Count Kit (Nacalai Tesque, Kyoto, Japan), according to the manufacturer's instructions. 


\section{ATP Assay}

The left ventricular samples obtained at the ends of the global ischemia and reperfusion periods were frozen immediately in liquid nitrogen and stored at $-70^{\circ} \mathrm{C}$ until further analysis, as described previously. ${ }^{14}$ ATP assay was performed with an ATP Bioluminescence Kit (Promega, Madison, Wis).

\section{Mitochondrial Swelling Assay}

To examine the effect of VS on calcium-induced PTP opening, we isolated mitochondria from the left ventricular tissues at ex vivo reperfusion and measured the swelling with a spectrophotometer, as described previously. ${ }^{15}$

\section{DePsipher Assay of Mitochondrial Transmembrane Potential}

To examine the effect of $\mathrm{ACh}$ on the mitochondrial transmembrane potential of hypoxia-reoxygenated cardiomyocytes, we used a DePsipher Mitochondrial Potential Assay Kit (Trevigen, Gaithersburg, Md), as described previously. ${ }^{5}$

\section{Protein Preparation and Immunoblotting}

As described previously, ${ }^{4,5,14}$ the left ventricular samples obtained at the end of the reperfusion period in the ex vivo study were prepared for immunoblot analysis. Equal amounts of proteins $(50 \mu \mathrm{g}$ of total protein) were separated by means of sodium dodecylsulfate-polyacrylamide gel electrophoresis and transferred to a polyvinylidene difluoride membrane (Millipore, Temecula, Calif). After blocking nonspecific sites with 5\% nonfat milk in Tris-buffered saline supplemented with $0.1 \%$ Tween 20 overnight, the membranes were probed with primary antibodies against Akt (diluted 1:1000; Cell Signaling, Danvers, Mass), phospho-Akt (diluted 1:1000, Cell Signaling), Bcl-2 (diluted 1:1000; Santa Cruz Biotechnology, Santa Cruz, Calif), and phospho-Bad (diluted 1:1000; Oncogene, Cambridge, Mass). Anti-mouse and anti-rabbit immunoglobulin Gs conjugated with horseradish peroxidase (diluted 1:2000, Santa Cruz) were used as secondary antibodies and developed with an ECL chemiluminescence reagent (Amersham, Piscataway, NJ). As described previously, ${ }^{14}$ cytochrome $\mathrm{C}$ was detected by using the antibody against cytochrome C (diluted 1:1000, Santa Cruz Biotechnology) from the cytosolic and mitochondrial fractions.

\section{Caspase-3 Activity Assay for Detection of Apoptosis}

To assess the effect of VS on reperfusion-induced apoptosis, we measured caspase-3 activity with a Caspase-3/CPP32 Colorimetric Assay Kit (BioVision, Mountain View, Calif). ${ }^{5}$ The left ventricular samples from the ex vivo study were lysed, and caspase- 3 substrate was added to the cytosolic extract. Caspase activity was measured with a spectrophotometer (Ultrospec 3000; Pharmacia, Uppsala, Sweden).

\section{Statistical Analysis}

Nonparametric multiple-comparison tests among 3 or more groups were performed by using a Steel-Dwass test ${ }^{16,17}$ with Excel Statistics version 5.0 (Esumi). Values are expressed as the mean \pm standard deviation.

\section{RESULTS}

\section{Effect of VS and ACh on Reperfusion and Reoxygenation Injury}

VS reduced myocardial infarct size independent of its bradycardiac effects. As shown in Figure 1, the control group with a heart rate of 250 beats/min showed a small myocardial infarct size of $43 \% \pm 5 \%$. However, other control groups with heart rates of 300 and 350 beats/min had large infarct sizes of $85 \% \pm 9 \%$ and $88 \% \pm 7 \%$, respectively. In contrast, VS treatment reduced the myocardial infarct size irrespective of the heart rate $(37 \% \pm 4 \%$ for 250 beats $/ \mathrm{min}, 34 \% \pm 6 \%$ for 300 beats $/ \mathrm{min}$, and $39 \% \pm 4 \%$ for 350 beats/min), thus confirming that the myocardial protection provided by VS is independent of its bradycardiac effects. Therefore for the rest of the studies, we paced the heart at 300 beats/min with vagal nerve stimulation.

At a heart rate of 300 beats/min, the protective effects of VS on reducing the myocardial infarct size was abolished by treating the hearts with the PTP opener atractyloside before reperfusion, showing large myocardial infarction $(75 \% \pm$ 9\%). The PTP opener did not have any significant effect on the infarct size of the SS-treated hearts (Figure 1,C).

In vitro model. Although a hypoxia-reoxygenation challenge remarkably reduced the viable cell count (Figure 1, $D)$, ACh significantly improved cell survival $(23 \% \pm 2 \%$ vs $65 \% \pm 3 \%$ ). A treatment with atractyloside alone had no effect on cell viability; however, improvement of cell survival by ACh was significantly attenuated in the presence of atractyloside $(34 \% \pm 2 \%, P<.05$ vs ACh-treated cells $)$.

\section{Effect of VS on Functional Recovery of Reperfused Hearts After Global Ischemia}

In addition to the anti-infarct effect, VS exerted a beneficial effect on functional recovery of reperfused hearts after global ischemia (Table 1). When compared with the SS-treated left ventricle, the VS-treated left ventricle showed significantly high performance throughout the 120-minute reperfusion period. At the end of the reperfusion period, the developed pressure of the VS-treated left ventricle reached almost twice that of the SS-treated left ventricle $(78 \pm 5$ vs $45 \pm 8 \mathrm{~mm} \mathrm{Hg}, P<$ .001). Such a beneficial effect of VS on functional recovery of the reperfused left ventricle from global ischemia was significantly attenuated by atractyloside administration into perfusate $(54 \pm 5 \mathrm{~mm} \mathrm{Hg}$ at the end of the reperfusion period, $P<.05$ vs the VS-treated left ventricle). Treatment with atractyloside alone did not significantly affect the developed pressure of the SS-treated left ventricle.

VS also significantly prevented a progressive increase in left ventricular end-diastolic pressure. At the end of the reperfusion period, the end-diastolic pressure of the SS-treated left ventricle became 3 times as high as that of the VS-treated left ventricle ( $38 \pm 4$ vs $12 \pm 2 \mathrm{~mm} \mathrm{Hg}$ ). The decreasing effect of VS on left ventricular end-diastolic pressure was almost abolished by atractyloside administration.

There was no significant difference in coronary flow among the 4 groups before ischemia (16 $\pm 2 \mathrm{~mL} / \mathrm{min}$ [SS], $17 \pm 3 \mathrm{~mL} / \mathrm{min}$ [VS], $16 \pm 4 \mathrm{~mL} / \mathrm{min}$ [SS with atractyloside], and $17 \pm 1 \mathrm{~mL} / \mathrm{min}$ [VS with atractyloside]) or during reperfusion $(12 \pm 3 \mathrm{~mL} / \mathrm{min}$ [SS], $13 \pm 2 \mathrm{~mL} / \mathrm{min}$ [VS], $11 \pm 5 \mathrm{~mL} / \mathrm{min}$ [SS with atractyloside], and $12 \pm 4$ $\mathrm{mL} / \mathrm{min}$ [VS with atractyloside]). 


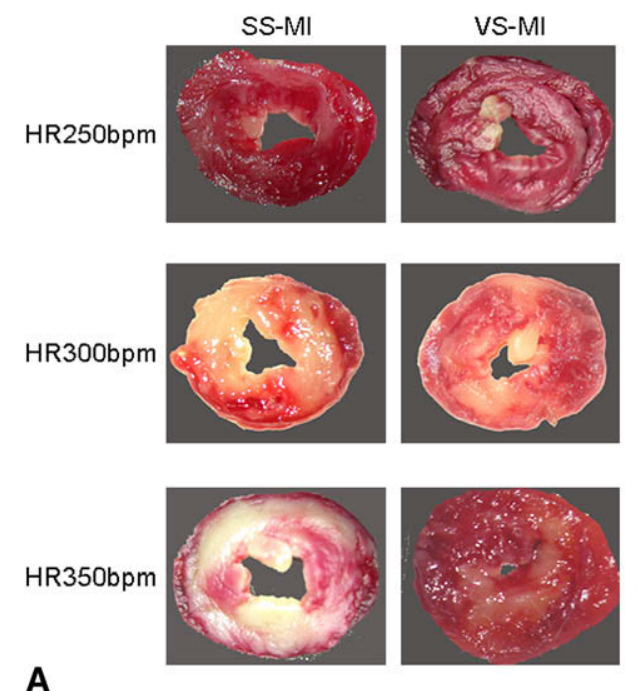

A

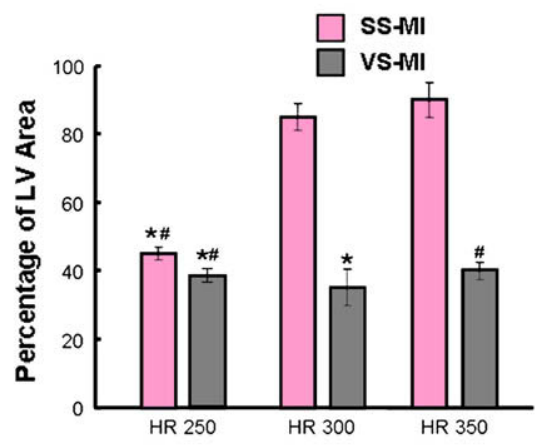

B
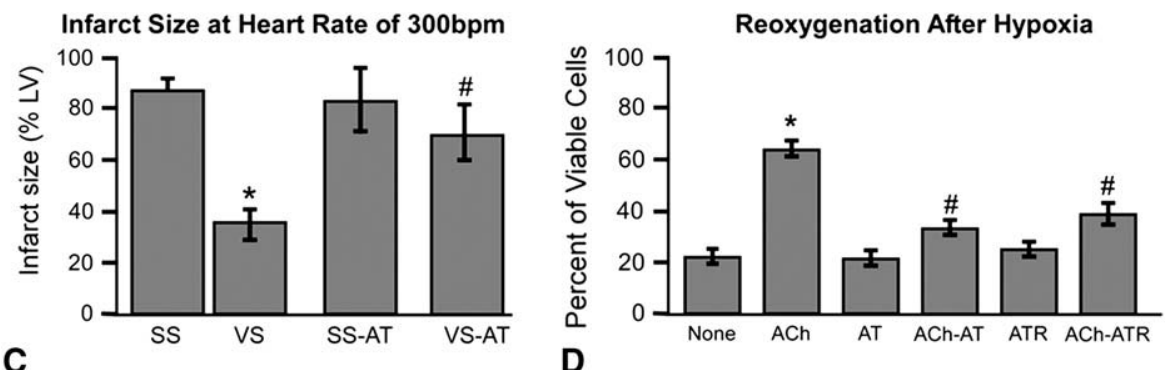

FIGURE 1. Effects of vagal stimulation on reperfused hearts after global ischemia and of acetylcholine on reoxygenated cardiomyocytes after chemical hypoxia. A, Representative left ventricular sections stained with triphenyltetrazolium chloride, with hearts paced at 300 beats/min ( $\mathrm{n}=10$ in each group). $H R$, Heart rate; SS, sham stimulation; $M I$, myocardial ischemia; VS, vagal stimulation. B, Quantitative analysis showing the percentage of infarct area in the hearts paced at different heart rates. $L V$, Left ventricular; $S S$, sham stimulation; $M I$, myocardial ischemia; $V S$, vagal stimulation. Values are expressed as the mean \pm standard deviation. ${ }^{*} P<.05$ from the sham stimulation-treated group with a heart rate of 300 beats/min; \#P<.05 from the sham stimulation-treated group with a heart rate of 350 beats $/ \min (n=10$ in each group). $C$, Graph showing an infarct size of the left ventricle treated with sham stimulation $(S S)$, vagal stimulation (VS), sham stimulation and atractyloside ( $S S$ - $A T$ ), and vagal stimulation and atractyloside (VS-AT). Each value in parentheses indicates the number of left ventricles ( $\mathrm{n}=10$ in each group). D, Graph showing a percentage of viable cells in untreated, acetylcholine-treated (ACh), atractylosidetreated (AT), ACh- and AT-treated (ACh-AT), atropine-treated (ATR), and ACh and atropine-treated (ACh-ATR) groups. For each treatment group, 5 culture dishes were analyzed. Values are expressed as the mean \pm standard deviation. $* P<.05$ from the sham stimulation-treated or untreated group; $\# P<.05$ from the vagal stimulation- or acetylcholine-treated groups.

\section{Effect of VS on Myocardial ATP Content at Global Ischemia-Reperfusion}

The VS-induced improvement of functional recovery of reperfused hearts seems to be involved in myocardial bioen- ergetics (Table 2). Global ischemia markedly reduced the myocardial ATP content. When compared with the SStreated left ventricle, the VS-treated left ventricle had a significantly high ATP content at the end of ischemia $(0.50 \pm$

TABLE 1. Left ventricular function during reperfusion period after 30 minutes of global ischemia

\begin{tabular}{|c|c|c|c|c|c|c|c|}
\hline \multirow{2}{*}{$\begin{array}{c}\text { Group } \\
\text { n }\end{array}$} & & \multicolumn{3}{|c|}{$\begin{array}{l}\text { Developed pressure (mm Hg) } \\
\text { Time after reperfusion }(\mathrm{min})\end{array}$} & \multicolumn{3}{|c|}{$\begin{array}{l}\text { End-diastolic pressure }(\mathrm{mm} \mathbf{H g}) \\
\text { Time after reperfusion }(\mathrm{min})\end{array}$} \\
\hline & & 5 & 60 & 120 & 5 & 60 & 120 \\
\hline SS & 10 & $9 \pm 2$ & $40 \pm 4$ & $45 \pm 8$ & $21 \pm 3$ & $35 \pm 4$ & $38 \pm 4$ \\
\hline VS & 12 & $10 \pm 1$ & $74 \pm 6^{*}$ & $78 \pm 5^{*}$ & $9 \pm 2 *$ & $15 \pm 3^{*}$ & $12 \pm 2^{*}$ \\
\hline SS-AT & 7 & $9 \pm 1$ & $43 \pm 10$ & $44 \pm 6$ & $21 \pm 4$ & $28 \pm 4$ & $37 \pm 3$ \\
\hline VS-AT & 10 & $10 \pm 2$ & $46 \pm 3 \dagger$ & $54 \pm 5 \dagger$ & $19 \pm 3 \dagger$ & $30 \pm 3 \dagger$ & $35 \pm 4 \dagger$ \\
\hline
\end{tabular}

Values are presented as the mean \pm standard deviation. $S S$, Hearts treated with sham stimulation; VS, hearts treated with vagal stimulation; $S S-A T$, hearts treated with sham stimulation and atractyloside; $V S-A T$, hearts treated with vagal stimulation and atractyloside. $* P<.05$ from the sham stimulation group; $\dagger P<.05$ from the vagal stimulation group. 
TABLE 2. Myocardial ATP content before ischemia and at the end of ischemia and reperfusion

\begin{tabular}{lccc}
\hline & \multicolumn{3}{c}{ ATP content $(\boldsymbol{\mu} \mathbf{m o l} / \mathbf{L}$ per gram wet weight $)$} \\
\cline { 2 - 4 } Group & Before ischemia & Ischemia & Reperfusion \\
\hline SS & $1.68 \pm 0.04$ & $0.22 \pm 0.01$ & $0.53 \pm 0.01$ \\
VS & $1.62 \pm 0.03$ & $0.50 \pm 0.07^{*}$ & $1.02 \pm 0.09^{*}$ \\
SS-AT & $1.61 \pm 0.02$ & $0.18 \pm 0.01$ & $0.54 \pm 0.05$ \\
VS-AT & $1.64 \pm 0.03$ & $0.47 \pm 0.06 \dagger$ & $0.58 \pm 0.03 \dagger$ \\
\hline
\end{tabular}

Values are presented as the mean \pm standard deviation ( $\mathrm{n}=5$ for each group). ATP, Adenosine triphosphate; $S S$, hearts treated with sham stimulation; $V S$, hearts treated with vagal stimulation; $S S-A T$, hearts treated with sham stimulation and atractyloside; $V S$-AT, hearts treated with vagal stimulation and atractyloside. $* P<.05$ from the sham stimulation group; $\uparrow P<.05$ from the vagal stimulation group.

0.07 vs $0.22 \pm 0.01 \mu \mathrm{mol} / \mathrm{L}$ per gram of wet weight) and exhibited a rapid restoration of ATP content during 120-minute reperfusion $(1.02 \pm 0.09$ vs $0.53 \pm 0.01 \mu \mathrm{mol} / \mathrm{L}$ per gram of wet weight). The VS-mediated effect on myocardial ATP content during ischemia-reperfusion was abolished by atractyloside. The administration of atractyloside alone had no significant effect on the myocardial ATP content.

\section{Effect of ACh on Collapse in Mitochondrial Transmembrane Potential of Reoxygenated Cardiomyocytes}

A mechanism for VS-induced improvement in the recovery of myocardial ATP content during reperfusion is demonstrated in Figure 2. When DePsipher-loaded cardiomyocytes were subjected to reoxygenation after hypoxia, the dye remained mostly in the cytoplasm and fluoresced green. In contrast, the dye in ACh-treated cardiomyocytes appeared to accumulate within mitochondria and fluoresced bright red. However, this ACh effect was blocked by atractyloside, whereas atractyloside alone had no effect. These results indicate that ACh prevented the mitochondrial potential collapse that resulted in myocardial ATP depletion. Treating the cells with atropine also inhibited the protective effects of $\mathrm{ACh}$, confirming that ACh acts through muscarinic receptors in cardiomyocytes.

\section{Effect of VS on Mitochondrial Swelling of \\ Reperfused Hearts After Global Ischemia}

A reperfusion-related mitochondrial injury through the PTP opening was accompanied by its structural change (ie, swelling). As shown in Figure 3, A, the hearts treated with VS during ischemia were insusceptible to such a mitochondrial injury. In mitochondria isolated from the SS-treated hearts, $\mathrm{Ca}^{2+}$ evoked a large decrease in spectrophotometric absorbance at $520 \mathrm{~nm}$, indicating mitochondrial swelling through the PTP opening (Figure 3,A). In contrast, mitochondria from the VS-treated hearts showed a significant resistance to $\mathrm{Ca}^{2+}$-induced swelling. Atractyloside attenuated the mitochondrial resistance of the VS-treated hearts to $\mathrm{Ca}^{2+}$-induced swelling; administration of atractyloside to the SS-treated
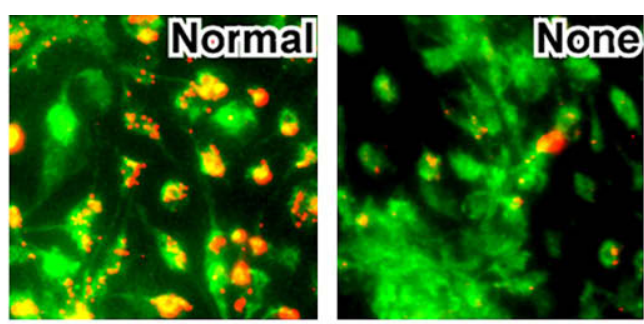

A
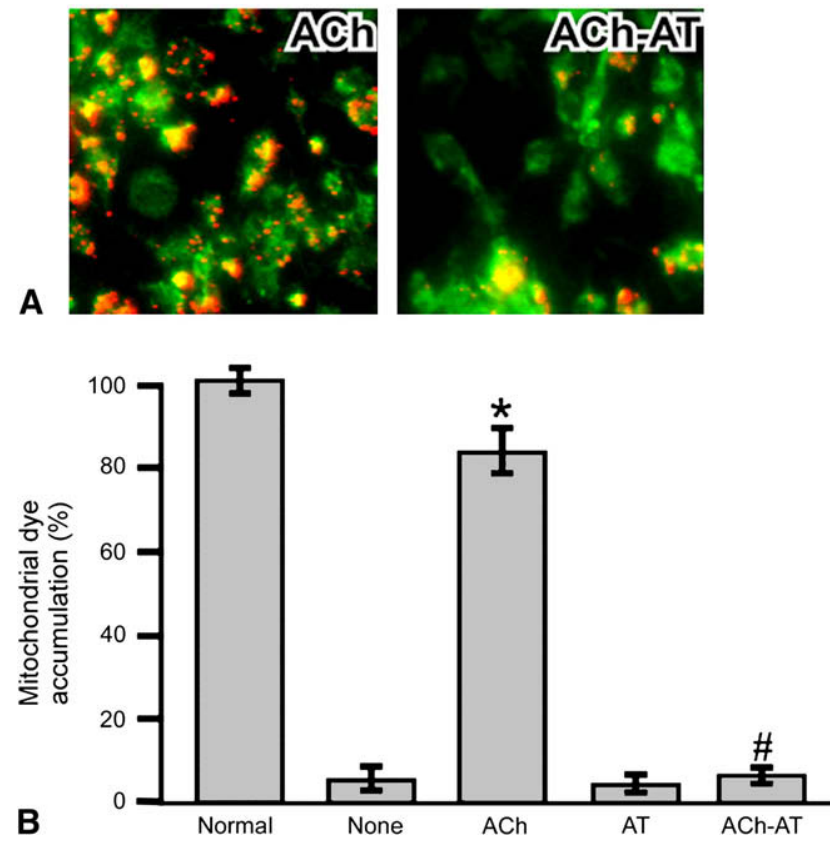

FIGURE 2. DePsipher assay in primary cultured rat cardiomyocytes. A, Representative confocal images of normal healthy cells and untreated (None), acetylcholine-treated ( $A C h$ ), and acetylcholine- and atractylosidetreated $(A C h-A T)$ cells at reoxygenation after hypoxia. B, Graph showing a percentage of cells with fluorescent red spots. For each treatment group, 5 culture dishes were analyzed. Values are expressed as the mean \pm standard deviation. ${ }^{*} P<.05$ from the untreated group; $\# P<.05$ from the acetylcholine-treated group.

hearts had no significant effect on $\mathrm{Ca}^{2+}$-induced mitochondrial swelling. These results are summarized in Figure 3, $B$.

\section{Effect of VS on Apoptosis-related Proteins}

VS-induced activation of antiapoptotic factors inhibiting the PTP at the reperfusion period, including Akt, Bad, and Bcl-2, is demonstrated in Figure 4. Even though the total amount of Akt remained unchanged, VS significantly upregulated a phosphorylated form of Akt when compared with that seen in the SS-treated hearts $(389 \% \pm 22 \%$ vs $75 \% \pm$ $10 \%)$. VS also upregulated the phosphorylated Bad $(159 \%$ $\pm 10 \%$ vs $23 \% \pm 4 \%$ ), thereby preventing its translocation into the mitochondria and inhibiting its binding to Bcl-2. Upregulation of these antiapoptotic factors by VS was significantly attenuated by atractyloside.

In the VS-treated hearts, as a consequence of upregulation of PTP-stabilizing proteins, such as Bcl-2, a release of 

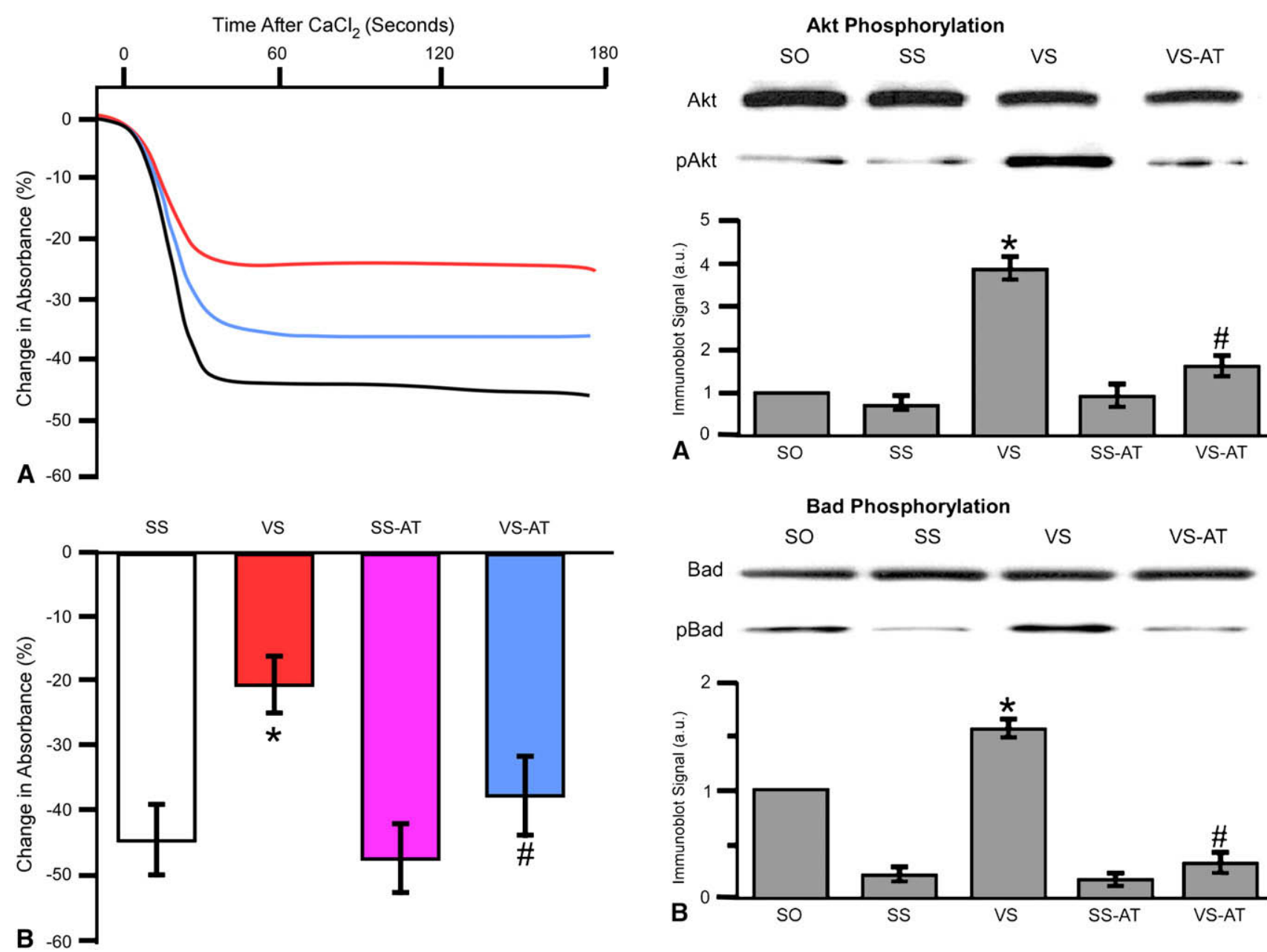

FIGURE 3. Mitochondrial swelling assay. The isolated mitochondria were incubated in medium containing $300 \mathrm{mmol} / \mathrm{L}$ sucrose and $10 \mathrm{mmol} / \mathrm{L} \mathrm{3-}$ morpholinopropanesulfonic acid ( $\mathrm{pH}$ 7.4) with Tris. After 1 minute, 1 $\mathrm{mmol} / \mathrm{L} \mathrm{Ca}^{2+}$ was added, and a decrease in absorbance caused by mitochondrial swelling was followed at $520 \mathrm{~nm}$, indicating mitochondrial permeability transition. A, Representative tracings of changes in spectrophotometric absorbance at $520 \mathrm{~nm}$ during a calcium challenge. B, Graph showing relative changes in the absorbance from baseline at 180 seconds after the calcium challenge. SS, Mitochondria isolated from sham-stimulated hearts; $V S$, mitochondria isolated from vagal-stimulated hearts; SS-AT, mitochondria isolated from hearts treated with sham stimulation and atractyloside; $V S-A T$, mitochondria isolated from hearts treated with vagal stimulation and atractyloside. Values are expressed as the mean \pm standard deviation ( $\mathrm{n}=5$ for each group). $* P<.05$ from the sham stimulation group; $\# P<$ .05 from the vagal stimulation group.

mitochondrial cytochrome $\mathrm{c}$ to the cytosol and an activation of caspase-3 during reperfusion were inhibited (Figure 5). However, atractyloside significantly attenuated these beneficial effects of VS on reperfused hearts.

\section{DISCUSSION}

The concept of reperfusion injury, although first reported in animal studies, is now recognized as a clinical problem
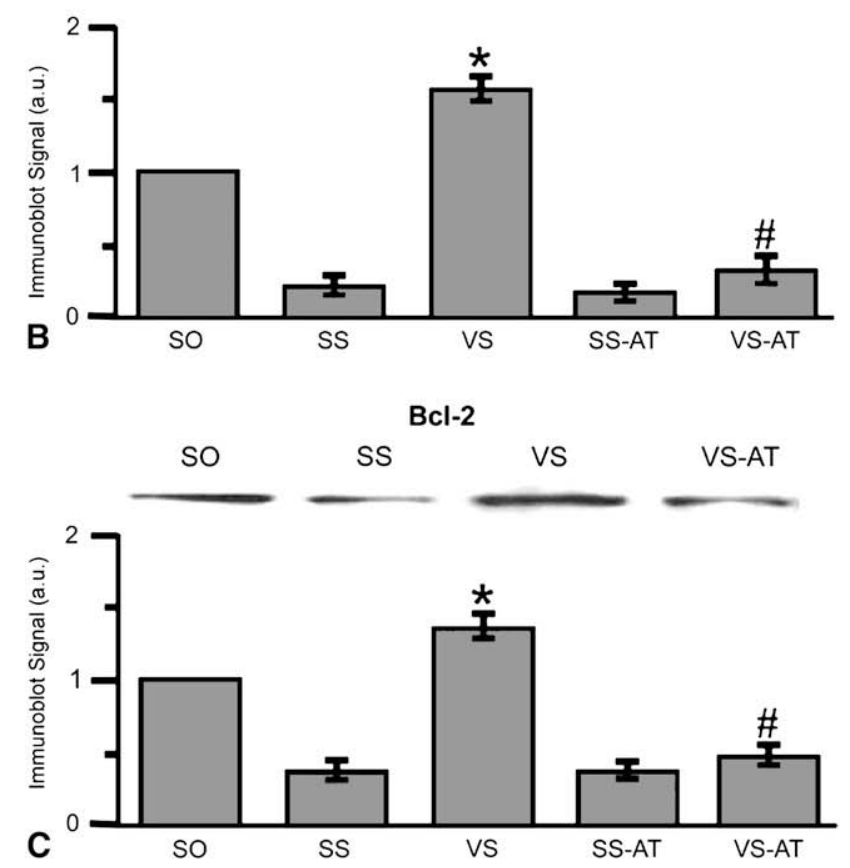

FIGURE 4. Effects of vagal stimulation on Akt phosphorylation (A), Bad phosphorylation (B), and Bcl-2 (C) of reperfused hearts. Each panel shows quantitative densitometric results of immunoblot analysis of left ventricles from sham-operated nonischemic hearts $(\mathrm{SO})$, ischemic hearts treated with sham stimulation ( $S S)$, ischemic hearts treated with vagal stimulation $(V S)$, ischemic hearts treated with SS and atractyloside (SS-AT), and ischemic hearts treated with vagal stimulation and atractyloside (VS-AT). Values are normalized by reference levels obtained from the sham-operated group and expressed as the mean \pm standard deviation ( $\mathrm{n}=5$ for each group). a.u., Arbitrary units. $* P<.05$ from the sham stimulation group; $\# P<.05$ from the vagal stimulation group. 

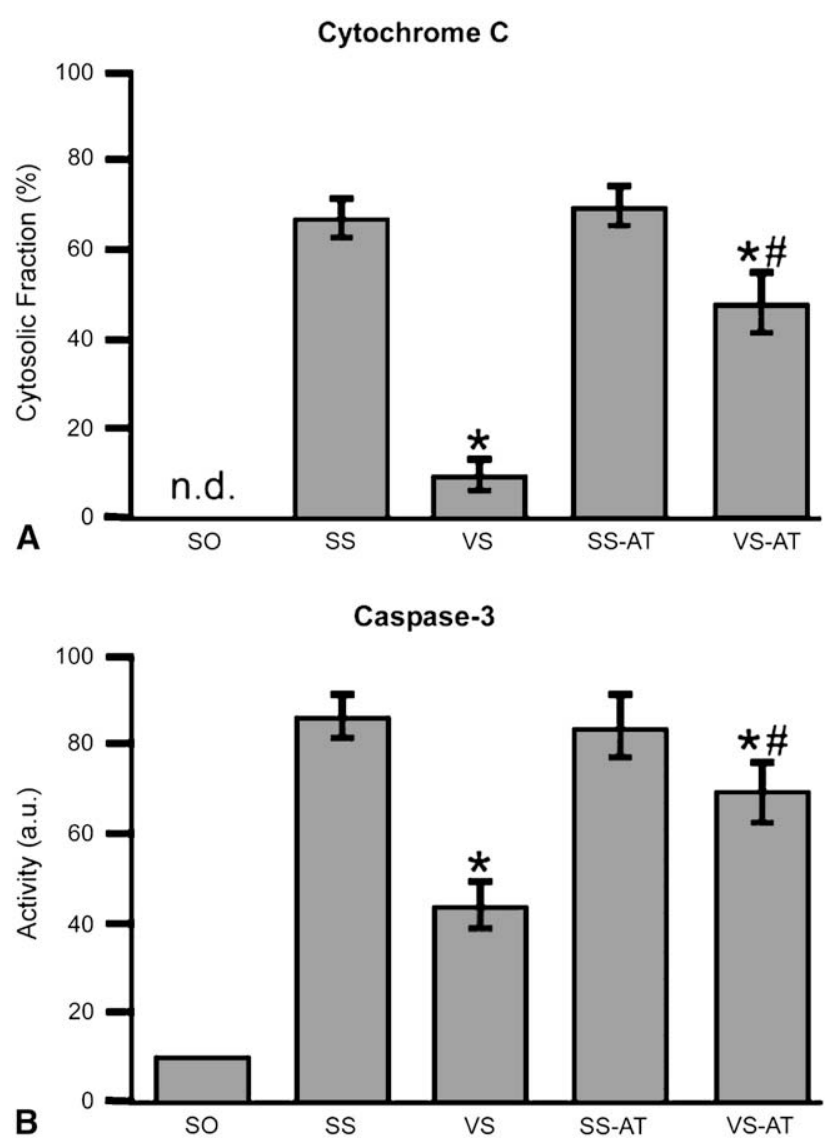

FIGURE 5. Effects of vagal stimulation on a percentage of cytosolic fractions of cytochrome c (A) and caspase-3 (B) activity of reperfused hearts. n.d., Not detectable; a.u., arbitrary units; $S O$, sham-operated nonischemic hearts; SS, ischemic hearts treated with sham stimulation; VS, ischemic hearts treated with vagal stimulation; $S S-A T$, ischemic hearts treated with sham stimulation and atractyloside; $V S-A T$, ischemic hearts treated with vagal stimulation and atractyloside. Values are expressed as the mean \pm standard deviation ( $\mathrm{n}=5$ for each group). $* P<.05$ from the sham stimulation group; $\# P<.05$ from the vagal stimulation group.

that results in microvascular damage, no-reflow phenomenon, and myocardial stunning and hibernation. ${ }^{7}$ The final consequence of this event is cardiac pumping dysfunction, leading to increased morbidity and mortality. In this study we have demonstrated a novel therapeutic strategy against acute ischemia-reperfusion injury using ex vivo and in vitro experimental models. The cardioprotective effects of VS during ischemia are evidenced as a reduction in infarct size, rapid restoration of myocardial ATP content, improvement of left ventricular dysfunction, activation of cell-survival signals, and inhibition of proapoptotic cascades, and all these effects were independent of its bradycardiac effects.

\section{VS During Ischemia Conditions the Myocardium Against Reperfusion Injury}

It is well known that the myocardium can be conditioned to acquire resistance to ischemia-reperfusion injury. Pre- conditioning before prolonged ischemia and postconditioning at reperfusion are inducible by brief episodes of intermittent ischemia and pharmacologic agents. ${ }^{7,18}$ Myocardial preconditioning is an option for adjuvant therapy against reperfusion injury at $\mathrm{CABG}$ and transplantation operations but not at coronary intervention for acute myocardial infarction. ${ }^{19}$ In the clinical setting of acute myocardial infarction, the onset of coronary occlusion is unpredictable; however, the start of reperfusion is predictable and controllable. ${ }^{20,21}$ Therefore myocardial postconditioning at reperfusion is an attractive strategy against ischemia-reperfusion injury. Zhao and associates ${ }^{9}$ first introduced the phenomenon of myocardial postconditioning. Although ischemic postconditioning has a dramatic effect on basic cardiology, a clinical application of ischemic postconditioning (ie, reintroduction of ischemia at the time of reperfusion) might be difficult. ${ }^{7}$ Recently, a more practical solution in clinical situations, pharmacologic postconditioning, has attracted much attention. ${ }^{22}$ However, there is now little information on its effectiveness in the clinical settings of acute myocardial infarction.

In addition to preconditioning and postconditioning, there might be a time window of therapeutic opportunity. Our previous study ${ }^{4}$ showed that VS protected hearts against ischemia-induced lethal arrhythmias by preserving gap-junction proteins, suggesting that the conditioning of the ischemic myocardium through manipulation of cellular and molecular functions is inducible by VS during coronary occlusion. We also demonstrated that ACh mimicked the beneficial effects of VS with an in vitro cellular model ${ }^{5}$ and speculated that VS increased an ACh release from its nerve endings, even at the ischemic area. Recently, using a microdialysis technique, Kawada and coworkers ${ }^{11}$ confirmed that VS increased the myocardial interstitial ACh level in the ischemic region approximately 20 times higher than the baseline level. These findings support that the potent cardioprotectant $\mathrm{ACh}$ released by VS can exert its beneficial effects on the ischemic myocardium.

\section{VS-induced Protection Independent of the Bradycardiac Effect}

Activation of vagal tone is known to induce bradycardia, and therefore it is possible that VS induces its protective effects through its bradycardiac effect. In our study bradycardia with a heart rate of $250 \mathrm{bats} / \mathrm{min}$ induced a significant reduction in the myocardial infarct size with or without VS treatment, whereas other groups with heart rates of 300 and 350 beats/min showed a large infarct size. Even though the exact mechanism behind the bradycardia-induced protection is not known, bradycardia has been suggested to improve the relationship between myocardial oxygen supply and demand, thus favorably redistributing the ischemic blood flow. ${ }^{23}$ In contrast, VS treatment exhibited a significant reduction in the myocardial infarct size irrespective of 
the heart rate, thus confirming that the protective effects induced by VS were independent of its bradycardiac effect.

\section{VS Suppresses Reperfusion-induced Opening of the PTP}

The beneficial effects of VS would be exerted through preservation of mitochondrial function and were blocked by the PTP opener atractyloside. The mitochondrial pathway of cell death has been widely demonstrated to be mediated by the opening of the PTP. Halestrap and colleagues ${ }^{24}$ demonstrated that pharmacologic inhibition of PTP opening mimicked the effects of ischemic preconditioning and postconditioning. With a human trabecula model, Shanmuganathan and associates ${ }^{3}$ also showed that PTP inhibitors improved contractile dysfunction at hypoxia-reoxygenation injury, supporting our results.

\section{VS Attenuates Reperfusion-related Apoptosis}

Green and Kroemer ${ }^{12}$ suggested a 3-step model for apoptotic cell death: a premitochondrial phase with activation of signal transduction cascades, a mitochondrial phase with permeabilization of the PTP, and a postmitochondrial phase with activation of apoptotic cascades by the proteins released from mitochondria to the cytosol. In the present study VS induced the phosphorylation of Akt and Bad, which are important factors on the premitochondrial cellsurvival cascades. The phosphorylation of Bad prevented its translocation to the mitochondria, thereby inhibiting its binding with $\mathrm{Bcl}-2$, which is essential for maintaining the PTP in a closed state. ${ }^{25} \mathrm{VS}$ also inhibited the release of mitochondrial cytochrome $\mathrm{c}$ into the cytosol and the activation of caspase-3. These results therefore provide evidence for the ability of VS to inhibit the 3 phases of apoptotic cell death.

\section{LIMITATIONS}

Our model of ex vivo heart perfusion with vagal nerve stimulation is not optimized to evaluate the recovery of the heart after cardioplegic arrest. Therefore in this study we used global ischemia to demonstrate the effects of vagal nerve stimulation to protect against reperfusion-induced injury. However, the future studies will be focused on simulating the exact clinical condition of cardioplegic arrest of the heart by using this model.

To adjust the intensity of stimulation at a given level in each heart by monitoring heart rate, we selected the right vagus for electrical stimulation. Therefore it is not clarified whether the electrical stimulation of the left vagal nerve can exert the same effectiveness as that of the right vagal nerve.

\section{CONCLUSIONS AND CLINICAL PERSPECTIVES}

Some elegant studies by Vinten-Johansen and colleagues ${ }^{26,27}$ have already demonstrated the protective effects of intermittent vagal nerve stimulation during off-pump CABG. In our study we have used much milder stimulation compared with that used in the previous study, and in addition to demonstrating the improvement in cardiac dysfunction, we also evaluated the molecular mechanism behind this protection. Therefore VS could be a potential candidate in preventing reperfusion injury in patients undergoing cardiac surgery.

Moreover, in medical conditions a thrombolytic therapy for acute myocardial infarction reduces mortality by as much as $30 \%$ when treatment is begun within 6 hours of symptom onset. ${ }^{28,29}$ Despite this impressive benefit, there is considerable experimental evidence suggesting that successful thrombolysis can be accompanied by reperfusion injury, an inflammatory event occurring within the reperfused myocardium that might cause additional injury and necrosis. With the results of our present study, a catheter-based intravascular VS therapy ${ }^{30}$ during coronary occlusion would be a clinically applicable method for delivering the potent endogenous cardioprotectant $\mathrm{ACh}$ to the ischemic myocardium before reperfusion.

We thank Ms Masayo Yamamoto for her kind technical assistance in immunoblotting and cell culture.

\section{References}

1. Ishii H, Ichimiya S, Kanashiro M, Amano T, Imai K, Murohara T, et al. Impact of a single intravenous administration of nicorandil before reperfusion in patients with ST-segment-elevation myocardial infarction. Circulation. 2005; 112:1284-8.

2. Wu ZK, Laurikka J, Saraste A, Kyto V, Pehkonen EJ, Savunen T, et al. Cardiomyocyte apoptosis and ischemic preconditioning in open heart operations. Ann Thorac Surg. 2003;76:528-34.

3. Shanmuganathan S, Hausenloy DJ, Duchen MR, Yellon DM. Mitochondrial permeability transition pore as a target for cardioprotection in the human heart. Am J Physiol Heart Circ Physiol. 2005;289:H237-42.

4. Ando M, Katare RG, Kakinuma Y, Zhang D, Yamasaki F, Muramoto K, et al. Efferent vagal nerve stimulation protects heart against ischemia-induced arrhythmias by preserving connexin43 protein. Circulation. 2005;112:164-70.

5. Kakinuma Y, Ando M, Kuwabara M, Katare RG, Okudela K, Kobayashi M, et al. Acetylcholine from vagal stimulation protects cardiomyocytes against ischemia and hypoxia involving additive non-hypoxic induction of HIF-1alpha. FEBS Lett. 2005;579:2111-8.

6. Murry CE, Jennings RB, Reimer KA. Preconditioning with ischemia: a delay of lethal cell injury in ischemic myocardium. Circulation. 1986;74:1124-36.

7. Yellon DM, Downey JM. Preconditioning the myocardium: from cellular physiology to clinical cardiology. Physiol Rev. 2003;83:1113-51.

8. Krieg T, Qin Q, Philipp S, Alexeyev MF, Cohen MV, Downey JM. Acetylcholine and bradykinin trigger preconditioning in the heart through a pathway that includes Akt and NOS. Am J Physiol Heart Circ Physiol. 2004;287: H2606-11.

9. Zhao ZQ, Corvera JS, Halkos ME, Kerendi F, Wang NP, Guyton RA, et al. Inhibition of myocardial injury by ischemic postconditioning during reperfusion: comparison with ischemic preconditioning. Am J Physiol Heart Circ Physiol. 2003;285:H579-88.

10. Hausenloy DJ, Yellon DM. New directions for protecting the heart against ischaemia-reperfusion injury: targeting the Reperfusion Injury Salvage Kinase (RISK)pathway. Cardiovasc Res. 2004;61:448-60.

11. Kawada T, Yamazaki T, Akiyama T, Li M, Ariumi H, Mori H, et al. Vagal stimulation suppresses ischemia-induced myocardial interstitial norepinephrine release. Life Sci. 2006;78:882-7.

12. Green DR, Kroemer G. The pathophysiology of mitochondrial cell death. Science. 2004;305:626-9. 
13. Miyano H, Nakayama Y, Shishido T, Inagaki M, Kawada T, Sato T, et al. Dynamic sympathetic regulation of left ventricular contractility studied in the isolated canine heart. Am J Physiol Heart Circ Physiol. 1998;275:H400-8.

14. Rajesh KG, Sasaguri S, Ryoko S, Maeda H. Mitochondrial permeability transition-pore inhibition enhances functional recovery after long-time hypothermic heart preservation. Transplantation. 2003;76:1314-20.

15. Yamamura T, Otani H, Nakao Y, Hattori R, Osako M, Imamura H. IGF-I differentially regulates Bcl-xL and Bax and confers myocardial protection in the rat heart. Am J Physiol Heart Circ Physiol. 2001;280:H1191-200.

16. Steel RGD. A rank sum test for comparing all pairs of treatments. Technometrics. 1960;2:197-207.

17. Dwass M. Some k-sample rank-order tests. In: Olkin I, ed. Contributions to Probability and Statistics. Stanford: Stanford University Press; 1960:198-202.

18. Kloner RA, Rezkalla SH. Preconditioning, postconditioning and their application to clinical cardiology. Cardiovasc Res. 2006;70:297-307.

19. Tomai F, Crea F, Chiariello L, Gioffrè PA. Ischemic preconditioning in humans: models, mediators, and clinical relevance. Circulation. 1999;100:559-63.

20. Laskey WK. Brief, repetitive balloon occlusions enhance reperfusion during percutaneous coronary intervention for acute myocardial infarction. Catheter Cardiovasc Interv. 2005;65:361-7.

21. Staat P, Rioufol G, Piot C, Cottin Y, Cung TT, L'Huillier I, et al. Postconditioning the human heart. Circulation. 2005;112:2143-8.

22. Philipp S, Yang XM, Cui L, Davis AM, Downey JM, Cohen MV. Postconditioning protects rabbit hearts through a protein kinase $\mathrm{C}$-adenosine $\mathrm{A} 2 \mathrm{~b}$ receptor cascade. Cardiovasc Res. 2006;70:308-14.
23. O'Brien P, Drage D, Saeian K, Brooks HL, Warltier DC. Regional redistribution of myocardial perfusion by UL-FS 49, a selective bradycardic agent. Am Heart J. 1992;123:566-74.

24. Halestrap AP, Kerr PM, Javadov S, Woodfield KY. Elucidating the Molecular mechanism of the permeability transition pore and its role in reperfusion injury. Biochim Biophys Acta. 1998;1366:79-94.

25. Murphy E. Primary and secondary signaling pathways in early preconditioning that converge on the mitochondria to produce cardioprotection. Circ Res. 2004 94:7-16.

26. Ronson RS, Puskas JD, Thourani VH, Velez DA, Bufkin BL, Glass J, et al. Controlled intermittent asystole cardiac therapy induced by pharmacologically potentiated vagus nerve stimulation in normal and hibernating myocardium. Ann Thorac Surg. 2003;75:1929-36.

27. Bufkin BL, Puskas JD, Vinten-Johansen J, Shearer ST, Guyton RA. Controlled intermittent asystole: pharmacologic potentiation of vagal-induced asystole. Ann Thorac Surg. 1998;66:1185-90.

28. Gruppo Italiano per lo Studio della Streptochinasi nell'Infarto Miocardico (GISSI). Effectiveness of intravenous thrombolytic treatment in acute myocardial infarction. Lancet. 1986;1:397-402.

29. The GUSTO Investigators. An international randomized trial comparing four thrombolytic strategies for acute myocardial infarction. $N$ Engl J Med. 1993 329:673-82.

30. Schauerte P, Mischke K, Plisiene J, Waldmann M, Zarse M, Stellbrink C, et al Catheter stimulation of cardiac parasympathetic nerves in humans: a novel approach to the cardiac autonomic nervous system. Circulation. 2001;104:2430-5. 